\title{
Applying Chatbots to the Internet of Things: Opportunities and Architectural Elements
}

\author{
Rohan $\operatorname{Kar}^{1}$ \\ Hyderabad, \\ India
}

\author{
Rishin Haldar ${ }^{2}$ \\ School of Computing Sciences and Engineering, \\ VIT University, \\ Vellore, India
}

\begin{abstract}
Internet of Things (IoT) is emerging as a significant technology in shaping the future by connecting physical devices or things with the web. It also presents various opportunities for the intersection of other technological trends which can allow it to become even more intelligent and efficient. In this paper, we focus our attention on the integration of Intelligent Conversational Software Agents or Chatbots with IoT. Prior literature has covered various applications, features, underlying technologies and known challenges of IoT. On the other hand, Chatbots are a relatively new concept, being widely adopted due to significant progress in the development of platforms and frameworks. The novelty of this paper lies in the specific integration of Chatbots in the IoT scenario. We analyzed the shortcomings of existing IoT systems and put forward ways to tackle them by incorporating chatbots. A general architecture is proposed for implementing such a system, as well as platforms and frameworks - both commercial and open source - which allow for the implementation of such systems. Identification of the newer challenges and possible future research directions with this new integration have also been addressed.
\end{abstract}

Keywords-Internet of Things; Chatbots; Human-Computer Interaction; Conversational User Interfaces; Software Agents

\section{INTRODUCTION}

The Internet of Things (IoT) is not just a well-recognized phenomenon but one that is shaping the digital age. It introduces an era of interconnected smart objects or 'things' developed upon existing Internet architectures. By using unique addressing schemes and standard communication protocols, IoT interconnects these things or objects thereby creating a varied range of technologies that can interact with each other and reach common goals [1].

An essential goal of connecting various sensors, actuators and services and processing data from them is to generate situational awareness and enable machines and human users to make sense of themselves and their surrounding environments.

The proliferation of IoT can be seen through the adoption of these "smart devices" in our daily life which include applications in Manufacturing, Agriculture, Medical and Healthcare, Transportation, Building and Home Automation and Energy Management among others. A report by Gartner estimates that there will be over 20 Billion connected things in activity by 2020 with Cisco estimating the number to be over
50 Billion [2, 3]. Among them more than half of all IoT endpoints in the consumer space alone. Hence IoT is a phenomenon which is certain to play a major role in our daily interaction with the digitally connected world.

\section{A. Scope of Internet of Things}

Literature presents various ways to define the Internet of Things. The RFID group defines Internet of Things as "worldwide network of interconnected objects uniquely addressable, based on standard communication protocols." ITU [4] defines it as "a global infrastructure for the information society, enabling advanced services by interconnecting (physical and virtual) things based on existing and evolving interoperable information and communication technologies."

While considering the broad vision of IoT, this paper focuses on the perspective of connected things and its applications. To do this, we simply create a separation of concern between the fragmented lower Open System Interconnection (OSI) layers of IoT and the unified adopted upper layers of IoT communication which use the World Wide Web and its standard network protocols.

The entire IoT system consists of Sensors (such as temperature, light, and motion), Actuators (such as displays, sound and motors), Computation (programs and logic), and Communication interfaces (wired or wireless). However, based on established advantages presented in prior literature $[5,6,7,8]$, our scope will be limited to interaction with IoT through Web Application Programming Interfaces (API) and in particular Hypertext Transfer Protocol (HTTP) based Representational State Transfer (REST) Architectures. A popular approach to Web of Things has been illustrated in Fig 1 based on [6].

The Evans Data Corporation (EDC) Report: Internet of Things - Vertical Research Service study [9] reveals that more than half of IoT developers connect to devices primarily through the cloud. The massive growth and acceptance of these cloud-based platforms such as IBM IoT Platform, Amazon Web Services IoT, Microsoft Azure IoT and Cisco IoT are indicative of its popularity among IoT companies. Hence, this paper also proposes the use of IoT cloud-based platforms in the proposed system design. This is discussed further in Section 4. 


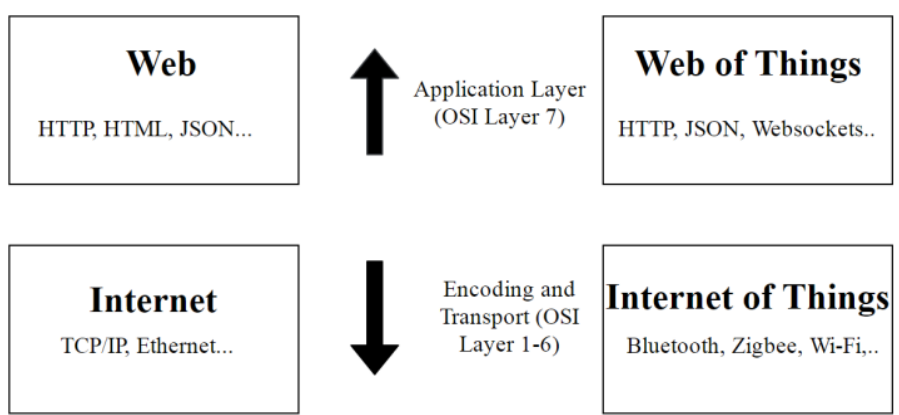

Fig. 1. Web of Things as shown in [6]

\section{B. Scope of Chatbots}

This paper proposes the use of Intelligent Conversational Agents. We refer to these as simply Chatbots (also known as Chatterbots or bots in general). Interestingly, there are many definitions for Chatbots in close relation with Software Agents (SA), Virtual Agents (VA) or Intelligent Personal Assistants (IPA) in literature and these have often been used in conjunction with each other. The term "Agents" itself has many definitions but among the earliest and most well-known uses of the term is [10] - "A self-contained, interactive and concurrently-executing object, possessing internal state and communication capability."

Software Agents can be most closely associated with Chatbots and has been well documented in prior literature [11]. The following key properties have been related to Software Agents [12]: (1) reactive, (2) proactive and goaloriented, (3) deliberative (4) continual (5) adaptive (6) communicative, and (7) mobile. However, the purpose of this paper is not to explore the various types of Software Agents and agent-based systems or its properties but rather propose the solution to challenges faced in IoT through the use of the umbrella term for these Intelligent Conversational Agents, Software Agents or Chatbots as we refer to them. It is also important to note that Software Agent distinguishes itself from Intelligent Agents (also known as Rational Agents). Intelligent agents are not only computer programs. They can also be machines, humans or anything that is capable of a goal directed behavior [13].

Typically Chatbots are classified into two types: (1) Chatbots that function based on Rules (2) Chatbots that function based on Artificial Intelligence (AI). Chatbots that function on rules are often limited as they are only as smart as they are programmed. On the other hand, AI based Chatbots give the impression of being "intelligent" as they are capable of understanding natural language, not just pre-defined commands and get smarter as they interact more due to their ability to maintain states. Based on this, concepts such as Virtual Agents and Intelligent Personal Assistants (IPA) have come up, which use natural language processing, as well as speech recognition techniques. For example, Apple Siri, Amazon Alexa, Microsoft Cortana and Google Assistant are some of the popular IPAs.

In this paper, we present a novel paradigm combining these two disparate concepts of IoT and Software Agents in a single solution. However, the studies of these paradigms have largely been separate endeavors. We discuss how using chatbots as intelligent conversational interfaces can be used to address critical problems in IoT. We also propose a high-level conceptual architecture and discuss key architectural elements involved in communicating with an IoT system through Chatbots. To explain in the context of real-world applicability, we put forth existing solutions to each of the components in the architecture including frameworks, platforms and specify open-source tools which can be used to build such a system.

The remaining paper is organized as follows: In section II we discuss our motivation for introducing this novel concept of Chatbots in Internet of Things and discuss other literature work that has helped shape this idea. In Section III, we evaluate and examine the shortcomings and challenges of current IoT systems and the opportunity for chatbots to address them. Section IV proposes a system design and the key architectural elements. Finally, we present our concluding remarks by assessing opportunities and scope for future research and development in Section V.

\section{Motivation AND BACKGROUND STUDY}

The key to the massive adoption and diffusion of IoT is the proliferation of Internet in our daily lives. We use the internet to search for information, check emails, consume media, and connect with people via social networks and so much more. With around $40 \%$ of the global population (3.4 Billion) currently using the world wide web, this number is estimated to increase to 7.6 billion global internet users in 2020, a majority of which use mobile devices (such as phones, tablets, and wearables) [14]. Hence the internet has played a vital role as a global backbone for information sharing and interconnection of physical objects with computing/networking capabilities for applications and services spanning numerous use cases. The Internet alone, however, cannot address all issues of IoT. First, we will briefly discuss the challenges in IoT, and then mention the motivation for choosing intelligent conversational interfaces.

\section{A. Challenges in IoT}

Despite the wide scale efforts to popularize IoT, it still offers many practical challenges. Primarily, IoT systems operate in isolated technology or vendor specific silos which inhibit capability, value, and interoperability and create a widely disparate area [15]. Specifically, by restricting heterogeneous devices (such as home appliances, mobiles, tablets, embedded devices), sensors and services to communicate with each other across interconnected networks, possibilities of countless applications are hindered.

Secondly, the sheer number of actively connected things has already started to create problems in application, device and data management in IoT [16]. To address this issue, IoT platforms (such as IBM Watson IoT, Microsoft Azure IoT, AWS IoT) offer scalable, distributed cloud-based services to allow businesses to connect to an established infrastructure service or software quickly, without being concerned about backend complexities. While IoT Cloud platforms are a step in the right direction, offering many advantages, it still presents many challenges particularly in interoperability which has led to the issues of platform fragmentation $[17,18]$. 
IoT systems also face a challenge of unifying User Interfaces (UI). It becomes increasingly difficult for users to keep track and access multiple applications, dashboards for every new "thing" in their ecosystem [19]. Hence unifying interfaces across multiple connected things and providing them with a high degree of smartness for improved user experience is a key challenge.

\section{B. Relevance of Chatbots}

According to a Business Insider study, Instant Messaging (IM) platforms (Such as Facebook Messenger, Slack, WhatsApp, and Telegram) have more active users than any other internet application including social networks, mailing applications etc. The same report shows that the top ten messaging platforms alone account for nearly 4 Billion users [20]. The global acceptance of chat based interfaces allows for ease of adoption and diffusion of newer technologies (such as Chatbot Applications) to be built on top of the pre-existing platforms. Therefore the global proliferation of chat as a Conversational User Interface (CUI) only furthers the motivation to develop interesting applications and use cases with chatbots.

Secondly, advancements made in the areas of Artificial Intelligence (AI), especially Natural Language Processing (NLP) have furthered the efficiency and quality of Chatbots allowing the user to make complex requests through simple natural language.

Finally, RESTful API's have been an important factor in the adoption of both IoT and Chatbots. The rationale behind it has been based on some of the following observations:

1) Ease of Development: Developers can take an API or service-oriented approach to development for both IoT as well as Chatbots. This means that application development methodologies would be the same with both embedded devices as with any web service (including Chatbots) that use Web APIs and in particular using RESTful architectures.

2) Ease of Deployment: Chatbot applications just like IoT applications can be designed and deployed on cloud platforms where developers need not be concerned about the underlying technologies such as Network, Storage, and Processing.

3) Standardized Web protocols: Owing to HTTP RESTful standards and protocols, it becomes technologically feasible and straightforward to integrate chatbot applications into IoT systems using application layer as the only concerned medium.

This ease of integration is a key motivation to develop platforms and frameworks which can synchronize chatbot applications within IoT platforms and frameworks.

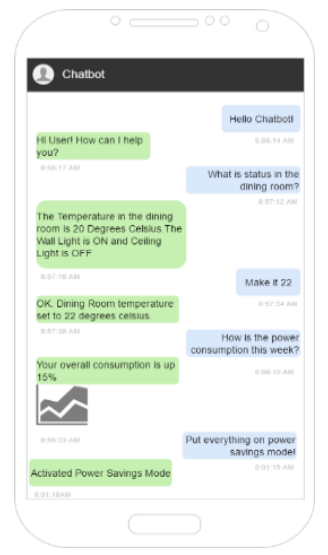

Fig. 2. A Sample User-Chatbot conversation

\section{OPPORTUNITIES FOR CHATBOTS IN IOT}

The shortcomings of modern IoT systems can be broadly classified into two types: (1) Technology Centric Challenges and (2) Human Centric Challenges. With the help of example chatbot-user conversations given in Fig. 2, we discuss the opportunities for Chatbots and demonstrate ways in which Chatbots can overcome challenges in IoT.

\section{Use Case (A)}

User: "Make the living room temperature comfortable."

Chatbot: "Since the weather outside is 17 degrees Celsius,

I am setting the living room temperature to 21.4 degree Celsius."

Use Case (B)

User: "How far is my car charged?"

Chatbot: "The Tesla Model S is currently 40\% charged. 3 Hours 10 minutes to full charge."

Use Case (C)

User: "Which lights are on?"

Chatbot: "The Guest Bedroom and Living room lights are ON."

User: "turn them off"

Chatbot: "The Guest Bedroom and Living room lights are now OFF."

Use Case (D)

User: "Help me setup my new device."

Chatbot: "Which device would you like to configure?

1) Smart Lock 2) Smart Kettle 3) Smart light?"

User: 1

Chatbot: "Ok, Enter your secret passcode for the smart lock." User: "******"

Chatbot: "Done. Smart Lock is now setup and ready for use."

Use Case (E)

Chatbot: "The monitoring service indicates that the smart lock has been offline for over 24 hours."

Chatbot: "Would you like me to report the issue to the Smart Lock Customer Support?"

User: "No, I want to talk to a human."

Human-Operator: "I can see the issue you are facing. I will try to resolve it remotely." 


\section{A. Technology Centric Challenges of IoT}

1) Data Management: A key challenge in the realm of IoT is managing the vast amount of big data being generated, as IoT sensors are becoming easily affordable. Not only is the data produced by the sensors large but also diverse (varying in quality and type) and multimodal (e.g., temperature, light, sound, video) in nature. While data deluge is one challenge, drawing insights from the data and being able to present it in a timely, understandable way is a much larger challenge.

The well-known Knowledge Hierarchy also called the DIKW (Data, Information, Knowledge, and Wisdom) Pyramid can best illustrate the situation in the context of IoT [21,22]. As one moves up the pyramid, the data gets smaller but becomes harder to gain abstractions and perceptions (Knowledge), which is required to derive actionable intelligence (Wisdom). Hence Chatbots are attempting to solve the problems of data and information management by mainly addressing the upper layers of the DIKW pyramid.

a) Data Context: Processing and analyzing of IoT data today is often done through the many "big data" solutions in cloud platforms which offer storage and computing infrastructure to accomplish the task. These existing IoT cloud solutions are capable of handling various data source and transmission challenges. However, a major challenge of existing IoT systems is conveying data about the different interconnected devices (sensors and objects) back to the user in a simple human understandable way. This requires context, which can be achieved by enabling Chatbots to understand the true intent of the user query and process information from their environments. Moreover, Chatbots have access to a global network of information via the internet and can be easily programmed to retrieve information in real-time which can improve the context.

In practical terms, Chatbots simplify the way we consume information from multiple screens and heavy data and graphics to simple Conversational User Interfaces (CUI) capable of delivering highly contextual and intelligible information within the flow of the chat app itself. Achieving this high-level of abstraction can deliver actionable intelligence (wisdom) with domain and user knowledge to maximize the full potential of IoT. For example, in use case (A), the user utterance was relatively vague. However, the Chatbot could have used contextual information from Realtime temperature along with knowledge of historical user preferences to perform a specific action.

b) Information Retrieval: Modern IoT dashboards are often saturated with various metrics, data points, charts and tables making it difficult for users to find the required information. Chatbots can effectively solve this problem by responding quickly to direct queries with highly accurate information. By understanding the specific intent of the user, they limit the scope of information to be presented. In terms of the knowledge hierarchy, Chatbots perform lookup and abstraction on IoT data. For example, in use case (B) the query only asked for Battery Charge related information. The Chatbot performed a lookup and limited the response accordingly.
2) Device and Application Management: A fundamental challenge of IoT has been the fragmentation of technology $[17,18]$. Having application interoperability between heterogeneous devices from a single remote (mobile device or operation terminal) is especially uncommon. For example, a smart light and a Heating Ventilation and Air Conditioning (HVAC) system may belong to the same network and environment yet have different user control terminals which are mutually independent entities, unaware of each other nor able to communicate with each other.

Chatbots are built on IM platforms (such as Facebook Messenger and Slack) which support multiple different chatbot applications within itself. A single chatbot application is also capable of communicating with multiple IoT devices through unique HTTP REST APIs. Chatbots can thus act as a single interface for communication between single purpose devices (e.g., Controlling two smart lights), heterogeneous devices (e.g., Controlling an HVAC and a Smart Car) and even different IoT ecosystems (e.g., Controlling Smart home devices and Smart Retail devices) in the case of cloud-based IoT. For example, in the use cases above, the same chatbot is utilized to converse with multiple heterogeneous devices. Given the right permissions are available, it can even communicate with Public IoT devices.

3) Bridging Data across Platforms and Services: IoT platforms can be seen as software development environments which handle Device Management, Application Management, Connection Management, Dashboard, and Analytics. Owing to platform fragmentation [17, 18], sharing of data across platforms is still uncommon. One solution is to solve the issue at the application level by using third party services, which through APIs, can access data from each platform. For example, device data from an IBM IoT platform can be collected and processed by an analytics service along with sensor data from an Azure IoT platform thereby bridging the two data sources.

4) Search and Discoverability: A key attribute of IoT is the natural tendency of objects to be dispersed in the environment while being interconnected and identifiable at class-level (i.e. common information across the same class) or serial-level (i.e. unique to an individual object) [23].

Based on the permissions of the requester and the availability of the connected objects in the scope of the environment, IoT requires lookup and discovery services to find and control these objects effectively. Such services include the availability of sensors and actuators which the Chatbot would be able to retrieve from the entities and convey to the user at the appropriate times. In use case (C), the Chatbot was effectively able to find the active smart lights in the environment.

5) Monitoring and Reporting: From IoT wearables such as health monitoring devices to industrial sensors which convey information in real time, monitoring and reporting are the main aspects of IoT systems.

Chatbots can also be effectively used as monitoring services by integrating with solutions such as Application 
Performance Management (APM). Accessing data from various IoT systems is a key advantage which is unique to Chatbots in this scenario.

Similarly, Chatbot services can utilize its reporting services and present the abstracted information to the user in an actionable and timely manner. In use case (E), the chatbot was monitoring the availability of the smart lock.

\section{B. Human Centric Challenges of IoT}

Chatbots were created with the primary purpose of improving the human-computer user experience. As such, solving the user experience shortcomings of IoT systems can be a significant opportunity for chatbots. IoT, with its complex system of applications, sensors, actuators and services present a daunting challenge of gaining technical knowledge to interact with these various components. Hence exposing settings and configurations to users presents an obvious and unfriendly burden that is far from ideal.

1) Cognitive Burden: The technology landscape of IoT is quickly changing. As newer features and use cases are introduced, there is an added responsibility to educate the end users which can be burdensome for both the users and the developers of the system. Complicated systems cause difficulties in adoption and diffusion. As an assistive technology, chatbots can simplify the learning curve by the following ways:

a) Help Texts: IoT-enabled Chatbots can feature help texts which clarify the user request to ensure that the action performed is same as the one intended.

b) Feature Recommendation: Chatbots can recommend possible actions to the user which can be made more intelligent and context-aware depending on user preferences and the dynamics of the environment.

c) Automating Tasks: Chatbots are good at automating common cyclic, tasks and can perform certain actions such as monitoring availability of sensors (uptime, downtime) and others through routine API calls, Websockets or PublisherSubscriber methods.

d) Frequently Asked Questions: Feedback loops can be easily integrated within chatbots to aggregate most common queries, and this data can be used to improve the future Quality of Service(QoS).

As more use cases are discovered, chatbots can make the adoption and diffusion of IoT systems significantly easier and reduce the cognitive burden required to understand the functionalities of these systems.

2) User Interface Opportunities: Graphical User Interfaces (GUI) for IoT are largely functional in nature. While it achieves simplicity by displaying virtual switches, sliders, and buttons, it still has some shortcomings which Chat interfaces can solve: (1) Chat interfaces understand natural language which makes interaction with the system as simple as asking queries and receiving answers. There is no need for navigation of menus and finding the right icon/button to perform a task. (2) Chatbots use machine learning techniques to understand an individual user and can personalize the service to that user. In this way, chatbots can maintain the natural flow of the conversation as well. (3) They are also highly contextual interfaces and can understand the intent in the scope of the past interactions which is a unique feature of chatbots and speech-based systems. (4) CUI concern mostly textual information, therefore, simple $\log$ files can be maintained and consequently analyzed to make debugging easier.

3) Configuration Challenges: Apart from the knowledge required to adapt to the new systems and ease the cognitive burden, each IoT device has its unique setup and configuration in terms of software, network, firmware etc. As the number of different IoT devices increase, it becomes challenging and burdensome at best to navigate the interfaces of various applications and appropriately configure the system. Often technicians are involved in setting up and explaining the uses of the system.

Chatbots can guide and advise users on the right configurations for their system by creating step-by-step setup processes. This also reduces human effort involved in setting up the system. For example, a new device was configured in use case (D).

4) Lack of Automated Error Reporting: The distributed nature of most IoT systems implies that user report databases of IoT errors are spread across multiple organizations, Operating System (OS) vendors, Internet Service Providers, and device vendors which makes automated problem reporting a major challenge. Furthermore, users themselves are uncertain which organization to report the particular issue. Thus, various stakeholders in the system have a limited understanding of the actual nature of the problem and avoid sharing information with each other. Chatbots, in this scenario, can access these reported problems and by integrating other services, be able to not only retrieve information from the IoT system but send information to it. In Use Case (E), the chatbot identified the correct stakeholder to send the error.

5) Support Challenges: Remedying hardware and software issues in modern consumer IoT systems can be an irksome task. The recourse is to call the service provider for technical support or in many cases return the product. Either way, it is an unnecessary burden on the user as well as the support vendors in today's cost structure.

Smart Chatbots often have support services built into their functionality. Human-in-the-loop processes can be used to handle situations the Chatbot is not trained or authorized to perform, in real-time. In this manner, users need not go beyond the scope of the chatbot application to look for product support. Any software issue or hardware malfunction can be monitored, and Over the Air (OTA) software repairs can be performed. Chatbots can also be used to schedule technical repairs making it a convenient and fast solution to customer support [24]. In use case (E), a human operator was made to intervene. 


\section{SYSTEM DESIGN AND ARCHITECTURAL ELEMENTS}

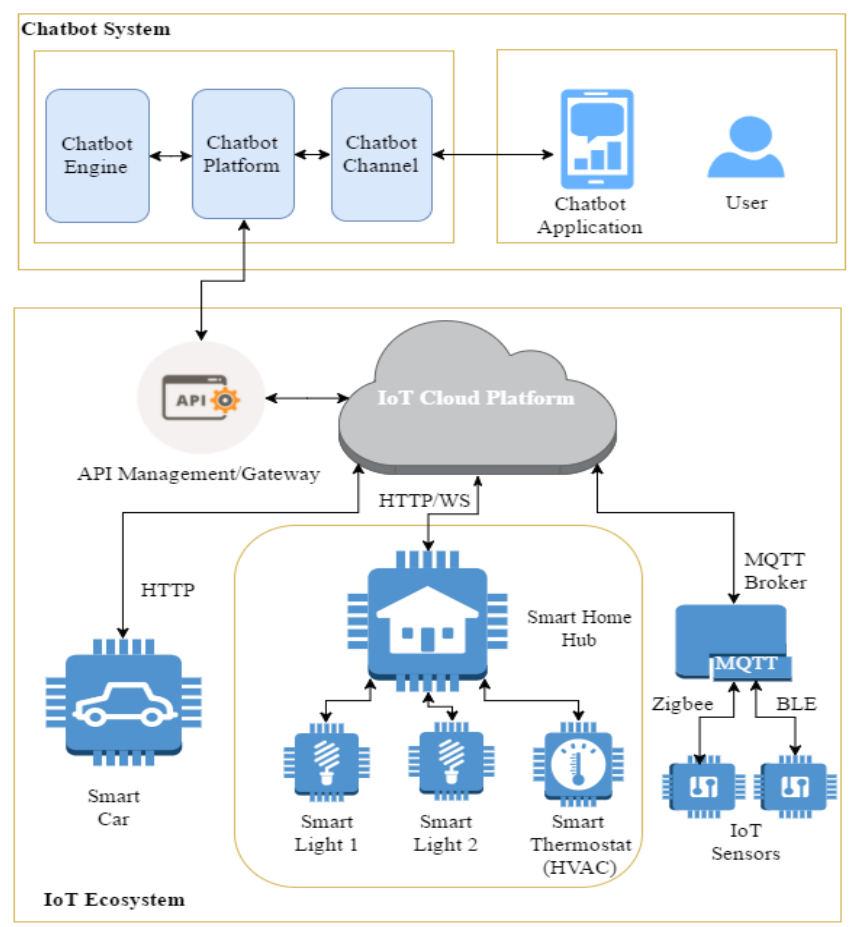

Fig. 3. Proposed System Design of IoT-Chatbot System

We present a conceptual system design which will aid in building Chatbot systems for IoT. Fig. 3 presents the highlevel view of the overall architecture consisting of the IoT system and the Chatbot system.

\section{A. IoT System}

1) IoT Devices: In the context of the paper, we refer to an IoT device in the broad scope of the term as a "uniquely identifiable IoT endpoint which can be accessed and controlled using RESTful Web APIs." In the situation an embedded device does not have APIs, there are existing solutions to create APIs for them easily. For Example, Using platforms such as Zetta, one can create cloud-based IoT systems with full-fledged API support. In this paper, we consider a Home automation system consisting of Smart lights (e.g., Philips Hue), Smart HVAC (comprised of a smart thermostat e.g., Nest) as well as a Connected Car or Smart Car (e.g., Tesla). However, in principle, any IoT device may be considered for interfacing with Chatbots.

2) IoT Cloud Platform: IoT Cloud based Platforms is an important enabling technology in many IoT systems today. They deal with various fragmented technologies in embedded devices from access protocols (e.g., Message Queuing Telemetry Transport (MQTT), Bluetooth Low Energy (BLE), HTTP etc.) to different services, Software Development Kits (SDK), and integrations. There have been positive reports on the established advantages of Cloud-based IoT platforms [25].

Our system design stresses on accessing and controlling the embedded devices in question, such as Smart Car, Light, Thermostat etc., through the API Management/Gateway of the IoT Cloud Platform, regardless of the standards and protocols of the individual embedded devices as seen through the representation of IoT sensors (which use Zigbee and BLE) connected to a MQTT broker which interfaces with the Cloud Platform. Similarly, the Smart home hub and the Smart Car use different protocols to interact with the IoT cloud platform with HTTP requests or Websockets. Popular IoT cloud platforms today include Microsoft Azure IoT, IBM IoT, APIGEE IoT and Cisco IoT.

\section{B. Chatbot System}

1) Chatbot Channels and Platforms: Chatbot Channels are applications which run Chatbots on supported Mobile devices (e.g., Smartphones, Tablets, smartwatches) or Terminals (e.g., Desktop Applications).They are typically built on top of the existing instant messaging platforms. Popular Chatbot channels include Facebook Messenger, Slack, Telegram, Kik, Skype, Line and Twilio SMS. These channels are essentially the Chatbot applications in which a user interacts with the bot. The area of Chatbot development is still in its infancy, and there can be many different architectural approaches in implementing Chatbots.

In some approaches, the channels are interfaced separately with the Chatbot Platforms through connectors. In Fig 3, the Chatbot Platforms are hosted on cloud services which may use Webhooks to communicate with the Channel. It is important to note in this scenario that we consider text-based Input/output (I/O) of Chatbots to IoT. However, by using SDKs it is possible to integrate IoT to voice/speech-based commands as seen in Intelligent Personal Assistants such as Amazon Echo (which uses Alexa SDK) and Google Home (which uses Google Assistant SDK).

2) Chatbot Engine: Perhaps the most important component of a Chatbot is the engine, often referred to as Natural Language Understanding (NLU) engine. It is responsible for translating natural language into machine understandable action. Chatbot engines are often highly complex, using various NLP models and ML techniques to provide acceptable levels of accuracy. To make it easier for Chatbot developers, many companies offer the processing capability of the Chatbot engine as a Software-as-aService(SaaS) or 'AI-as-a-service' which are applied to Chatbot applications using APIs. For example, Wit.ai and Microsoft LUIS.

This paper is primarily focused on listing the relevant key components of the engine and its functionality in the context of IoT, not on designing the NLP techniques for the Chatbot engine. Next, we include key concepts typically associated with chatbot engines [26, 27]:

- Entity Recognition: Entities are domain specific information extracted from the utterance that maps the natural language phrases to their canonical phrases to understand the intent. They help in identifying the parameters which are required to take a specific action. To train the chatbot engine, entities which are expected to give the same actions are typically grouped together. Common entities can be predefined as they can be used in many different scenarios. For example, 
Currency, Color, Date time, Location, Number etc. Domain specific entities can be trained to recognize similar phrases. IoT devices are one such domain specific entity.

For example, for the utterance: "Thermostat", the acceptable phrases may be trained as "Thermostat", "heat", "heating", "AC","air conditioning" and will be decoded as \{"type":iot, "device": "Thermostat"\} where the entity is IoT. Entities may also have its own attributes. Example 2: The utterance- "\$15" can be decoded as \{"type": "money", "amount":15, "currency": "dollars"\} where the entity is Money represented in JSON format.

- Context Determination: Determining the Context of the current user expression is an important feature of modern Chatbots. Understanding context can be important to handle situations where the utterances may be vague and have multiple meanings depending upon the history of the conversation. Contexts represent the ability of agents to maintain state (also called lifespan or the number of utterances after which the context will be removed) and match the requisite intent.

For example, if the user asks "Turn on the guest bedroom lights" asking about the bedroom room (location) in the first utterance and then in the following utterances, asks a typically vague statement such as: "turn it off" the Chatbot uses context to understand the second query relates to the earlier guest bedroom lights.

- Intent Extraction: Intents are the crux of conversational UI in chatbots. The intents represent what the users are looking to accomplish: get status updates, turn on/off devices, ask for help etc. The message passed from the user (utterance) in natural language is first analyzed for the intent. This implies, mapping a phrase to a specific action that should be taken by the IoT system as well as the specific dialog to be returned from the Chatbot. The information contained in an intent would be the context and action.

- Action Classification: Action refers to the steps that the IoT device will take when the intent of the user input is recognized. Actions have specified parameters which categorize details about it and triggered only when the intent recognizes them. For a smart home, the actions may be smartHome.lightsOn, smartHome.doorLock, smartHome.getStatus. In this scenario, other parameters may also be defined such as location (e.g., Dining room), time start/end (e.g., 10am, Thursday etc.), schedule (e.g., every hour, every minute) etc.

Once the action has been set and the minimum required parameters have also been defined, the right intent can be mapped to an IoT API endpoint. Meanwhile, the chatbot is required to maintain the natural flow of the conversation with the user. This is typically handled by a conversation module which models the semantics of the conversation. The quality of this conversation module varies from one Chatbot engine service to another, depending on the complexity of the AI.
Hence in this paper, we assume the bare minimum case of returning a simple, relevant response to the user without going into the conversation module.

Chatbots can also be built using existing frameworks simplify the end-to-end process of creating and integrating Chatbots into messaging and IoT platforms. The key advantages of using a Chatbot framework are: (1) Ease of Development from pre-defined actions, integrations and SDK support for various IoT systems (2) Ability to 'write once deploy anywhere' through integrations with multiple Chatbot Channels. (3) Ability to use AI-as-a-service. For example, LUIS.ai in the case of Microsoft Bot Framework. Other popular Chatbot frameworks include API.ai and IBM Watson Conversation Service

\section{FutURE RESEARCH AREAS AND CONCLUSION}

IoT is poised to become intrinsic to the day to day activities in the future. However, the dynamic nature of IoT has its share of difficulties, and this paper has put forward the concept of using Chatbots to address some of the challenges in IoT. Through this initial endeavor we can identify possible areas that can be worked on for the future which show great potential:

1) Stronger AI-powered Bots: As more advances are made in the field of AI, Software Agents will also grow to become more intelligent in the future. The goal of Strong AI has been to match the machine's intellectual capability to a human being. Immediate research challenges include improving decision-making ability to create more autonomous Chatbots. Better NLP as well as Natural Language Generation models will create more natural flows of conversation between humans and bots.

Most evidently, Chatbots will be able understand the opportunities in the conversation through improved context determination by seeking out knowledge from not only past history but a variety of external sources. Chatbots will also play a major role in the research areas of Intelligent Agents as well as Machine-to-Machine (M2M) research in IoT.

2) Cyber-Physical Systems and IoT: Cyber-Physical Systems refer to more advanced, next generation embedded Information and Communications Technology (ICT) systems. They share many similarities with IoT but with a higher combination and coordination of physical and computational elements [28]. The US National Science Foundation (NSF) identified cyber-physical systems among the key research areas in the foreseeable future [29]. IoT will play a major role in the transition to CPS as one of the key enabling technologies [16, 30]. Further advancements in AI aspects of Chatbots in IoT will be closely related to the Conversion, Cyber, Cognition and Configuration levels of the 5C CPS Architecture [31].

3) Wisdom of Things: The progress of Chatbots in IoT introduces the paradigm of feedback systems which has exciting research challenges in the areas of Wisdom of Crowds. The concept of wisdom of crowd suggests that aggregation of information can result in decisions that are 
better than what could have been achieved by any individual in the group [32]. In the context of IoT, the sharing of big data from billions of sensors and devices creates more value in the ecosystem as compared to not sharing. However, it requires data interoperability rather than simply accumulating multiple disparate data sources which are incompatible or have no similarities. Hence Chatbots in IoT systems can use techniques such as Human Swarming, an approach that uses real-time feedback loops from groups of users to make accurate insights. There are plenty of interesting research opportunities in acquiring accurate values from the crowd.

4) Evolution of the Semantic Web: As the Internet itself changes, there are many more opportunities for exciting research in the areas of IoT and Software Agents. The development of a Web 3.0 or Semantic Web and its impact on the future of Software Agents has been clearly described in the literature [33]. This presents a great opportunity for research in IoT regarding Semantic interoperability which can have a major impact on the IoT paradigm itself. The evolution of the Semantic Web will have a major impact on areas in pervasive computing, M2M technologies resulting in Software Agents being able to draw more value and achieve a higher level of wisdom than before.

Development in the field of IoT has been phenomenal in recent times. Similarly, Chatbot systems are also becoming more intelligent and sophisticated as the days progress. To the best of our knowledge, no work has been published detailing the specific integration of Chatbots to IoT. This paper has attempted to integrate these two fields together by enlisting the key architectural components required and envision possible ways to address some of the present challenges in IoT. We hope that this endeavor will lead to more intelligent, efficient and integrated IoT systems.

\section{REFERENCES}

[1] Atzori, L., Iera, A. and Morabito, G., 2010. The internet of things: A survey.Computer networks, 54(15), pp.2787-2805 J. Clerk Maxwell, A Treatise on Electricity and Magnetism, 3rd ed., vol. 2. Oxford: Clarendon, 1892, pp.68-73.

[2] Evans, D. "The Internet of Things How the Next Evolution of the Internet is Changing Everything (April 2011)." (2012): 346-360.

[3] van der Meulen, R., 2015. Gartner Says 6.4 Billion Connected 'Things' Will Be in Use in 2016, Up 30 Percent From 2015. Stamford, Conn

[4] ITU-T Recommendation database", ITU, 2016. [Online] Available:http://handle.itu.int/11.1002/1000/11559

[5] Guinard, D., Trifa, V., Mattern, F., \& Wilde, E. (2011). From the internet of things to the web of things: Resource-oriented architecture and best practices. In Architecting the Internet of Things (pp. 97-129). Springer Berlin Heidelberg.

[6] Guinard, Dominique; Vlad, Trifa (2015). Building the Web of Things. Manning. ISBN 9781617292682.

[7] Vermesan, O.,et al.., 2011. Internet of things strategic research roadmap. O. Vermesan, P. Friess, P. Guillemin, S. Gusmeroli, H. Sundmaeker, A. Bassi, et al., Internet of Things: Global Technological and Societal Trends, 1, pp.9-52

[8] Guinard, D., Ion, I. and Mayer, S., 2011, December. In search of an internet of things service architecture: REST or WS-*? A developers' perspective. International Conference on Mobile and Ubiquitous Systems: Computing, Networking, and Services (pp. 326-337). Springer Berlin Heidelberg
[9] Evansdata.com. (2016). Evans Data Corporation | Internet of Things Vertical Research Service. Available at: http://www.evansdata.com/reports/viewRelease.php?reportID=38

[10] Hewitt, C., 1977. Viewing control structures as patterns of passing messages. Artificial intelligence, 8(3), pp.323-364

[11] Nwana, Hyacinth S. "Software agents: An overview." The knowledge engineering review 11, no. 03 (1996): 205-244

[12] Schermer, Bart Willem. Software agents, surveillance, and the right to privacy: a legislative framework for agent-enabled surveillance. Leiden University Press, 2007

[13] Russell, Stuart Jonathan, Peter Norvig, John F. Canny, Jitendra M. Malik, and Douglas D. Edwards. "Artificial intelligence: a modern approach". Vol. 2. Upper Saddle River: Prentice hall, 2003.

[14] Broadband Commission, 2014. The state of broadband 2014: Broadband for all. Geneva, Switzerland: The United Nations

[15] S. Liang, "SensorThings API - connecting IoT devices, their location and theirdata,"2016.Available:http://www.eclipse.org/community/eclipse_ne wsletter/2016/march/article2.php

[16] Miorandi, D., Sicari, S., De Pellegrini, F. and Chlamtac, I., 2012. Internet of things: Vision, applications and research challenges. Ad Hoc Networks, 10(7), pp.1497-1516

[17] Celesti, Antonio, Maria Fazio, Maurizio Giacobbe, Antonio Puliafito, and Massimo Villari. "Characterizing Cloud Federation in IoT." In 2016 30th International Conference on Advanced Information Networking and Applications Workshops (WAINA), pp. 93-98. IEEE, 2016

[18] M. Wallace, "Fragmentation is the enemy of the Internet of Things | Qualcomm",Qualcomm, 2016

[19] M. Littman and S. Kortchmar, "The path to A programmable world," 2014.Available:http://footnote1.com/the-path-to-a-programmable-world/

[20] W. Mckitterick, "The Messaging App Report: How instant Messaging can be monetized," Business Insider

[21] Rowley, Jennifer E. "The wisdom hierarchy: representations of the DIKW hierarchy." Journal of information science (2007)

[22] Barnaghi, P., Wang, W., Henson, C. and Taylor, K., 2012. Semantics for the Internet of Things: early progress and back to the future. International Journal on Semantic Web and Information Systems (IJSWIS), 8(1), pp.1-21

[23] Bandyopadhyay, Debasis, and Jaydip Sen. "Internet of things: Applications and challenges in technology and standardization." Wireless Personal Communications 58, no. 1 (2011): 49-69

[24] Vinyals, Oriol, and Quoc Le. "A neural conversational model." arXiv preprint arXiv:1506.05869 (2015)

[25] Google, "Overview of Internet of things," Google Developers, 2016. Available: https://cloud.google.com/solutions/iot-overview

[26] Microsoft, "LUIS: Help," 2016. Available: https://www.luis.ai/Help

[27] API.ai,"Api.ai"2016. Available: https://docs.api.ai

[28] Rad, C.R., Hancu, O., Takacs, I.A. and Olteanu, G., 2015. Smart monitoring of potato crop: a cyber-physical system architecture model in the field of precision agriculture. Agriculture and Agricultural Science Procedia, 6, pp.73-79.

[29] Wolf, Wayne (November 2007). "The Good News and the Bad News (Embedded Computing Column".IEEE Computer. 40 (11): 104105.doi: 10.1109/MC.2007.404

[30] Wan, J., Chen, M., Xia, F., Li, D. and Zhou, K., 2013. From machine-tomachine communications towards cyber-physical systems. Comput. Sci. Inf. Syst., 10(3), pp.1105-1128

[31] Lee, J., Bagheri, B. and Kao, H.A., 2015. A cyber-physical systems architecture for industry 4.0-based manufacturing systems. Manufacturing Letters, 3, pp.18-23

[32] Joe Barkai. (2016). Wisdom of Things - Joe Barkai. Available at: http://joebarkai.com/wisdom-of-things

[33] Berners-Lee, T., Hendler, J. and Lassila, O., 2001. The semantic web. Scientific american, 284(5), pp.28-37 